\title{
APPROCHE HYDRODYNAMIQUE DE LA MICRODISTRIBUTION D'INVERTÉBRÉS BENTHIQUES EN EAU COURANTE
}

\author{
par H. Décamps', G. Larkouy² el D. 'Trivellato ${ }^{3}$.
}

La microdistribution des larves et nymphes de 3 espèces de simulies (Odagmia variegata, Odagmia obreptans et Simulium rupicolum) a été observée en divers cours d'eau pyrénéens.

Ces espèces se rencontrent en présence de vitesses importantes qui, dans les ruisseaux étudiés, étaient présentes dans les zones à écoulement torrential. Nous ne les avons pas rencontrées dans les zones à écoulement général de type fluvial. Les pierres lisses et d'un diamètre supérieur à $10 \mathrm{~cm}$ environ sont les substrats les mieux colonisés. Les faces amont faiblement inclinées présentent les plus fortes densités larvaires. Dans les zones à fort courant, en présence d'un gradient longitudinal même réduit des vitesses, les larves se concentrent dans une étroite bande où les vitesses sont maximales. Le corps des larves est couché vers l'aval et subit les pulsations du courant.

Les larves de simulies colonisent rapidement des canaux immergés dans un ruisseau (fig. 2); et par suite, les conditions hydrauliques locales peuvent être précisées aux endroits où se fixent les larves.

En une même station, le débit variant dans le temps, le type d'écoulement peut passer du fluvial au torrentiel (fig. 3 et 4).

Les profils des vitesses à la paroi, e'est-à-dire dans la zone où vivent les invertébrés, peuvent être déterminés en fonction de la rugosité apparente de la paroi (fig. 6).

Les conditions d'existence d'un film laminaire (sous couche laminaire) dépendent de la vitesse du courant et de la dimension des aspérités (fig. 7 et 8). Pour une vitesse de courant donnée, les relations entre l'épaisseur $\delta$ du film laminaire et la dimension $\varepsilon$ des aspérités déterminent les possibilités d'existence du film laminaire (fig. 8).

La variation de l'épaisseur du film laminaire est représentée en fonction de la vitesse du courant dans un abaque (fig. 9) qui tient compte également des conditions imposées par la dimension des aspérités.

Le film laminaire n'a qu'une influence réduite sur les conditions de vie des larves de simulies; la turbulence joue un rôle important sur ces conditions de vie.

\section{A hydrodynamic approach to the microdistribution of benthic invertebrates in running water.}

The microdistribution of larvae and pupae of three species of Simuliidae (Odagmia variegata, O. obreptans and Simulium rupicolum) has been observed in different streams in the Pyrenees.

1. Laboratoire d'Hydrobiologie, Université Paul-Sabatier, 118, route de Narbonne, 31077 Toulouse Cedex.

2. Laboratoire de Parasitologie, Université Paul-Sabatier, 37, allées JulesGuesde, 31 - Toulouse.

3. Institut de Mécanique des Fluides de l'ENSEEIH'T', Laboratoire associé au C.N.R.S., 31 - Toulouse. 
These species occur where there is a high water velocity which, in the streams studied, was present in zones with a torrential discharge. We have not found them in zones with a general discharge of the fluvial type. Smooth stones with a diameter greater than about $10 \mathrm{~cm}$ are the most frequently colonised substrata. The surfaces facing upstream and slightly inclined carry the greatest densities of larvae. In fast flowing zones with a longitudinal gradient of a reduction in velocites, the larvae are concentrated in a narrow band where the velocity is highest. The body of the larva lies in a downstream direction and moves with the pulsations of the current.

The larvae of Simuliidae rapidly colonise channels immersed in the stream (fig. 2); and therefore it is possible to define the hydrological conditions at the places where the larvae are attached.

The flow varies with time at the same station and the discharge can change from a fluvial type to a torrential type (fig. 3 and 4 ).

The profile of currents at the surface, that is to say in the zone where the invertebrates are living, can be determined as a function of the relative roughness of the surface (fig. 6).

The presence of a laminar film (laminar sub-layer) depends upon the strength of the current and the unevenness of the substratum (fig. 7 and 8 ). For a given current speed, the relationship between the thickness of the laminar film and the index of roughness or unevenness affects the possible formation of a laminar film (fig. 8).

The variation in the thickness of the laminar film is shown as a function of current speed in a graph ( $f i g$. 9) which also takes into account the conditions caused by index of roughness.

The laminar film has only a small influence on the living conditions of larvae of Simulidae whilst turbulence is an important factor.

\section{INTRODUCTION}

Les larves de simulies peuvent former d'abondantes populations dans les courants rapides. Elles figurent avec les blépharocérides parmi les insectes les plus résistants à la vitesse du courant (Dittmar 1955, Dorier et Vaillant 1955, Giudicelli 1968). Ainsi, parmi les espèces curopéennes, Odagmia ornata choisit-elle de préférence des courants compris entre 0,8 et $0,9 \mathrm{~m} / \mathrm{s}$ (Phillipson 1956 ) et Odagmia monticola et variegata des courants compris entre 1 et $2 \mathrm{~m} / \mathrm{s}$ (Phillipson 1957).

A ces vitesses, il n'est pas possible de faire appel, dans les conditions naturelles, à l'existence d'une couche limite pour interpréter la localisation des larves (Trivellato et Décamps 1968, Décamps et al. 1972). Des notions plus réalistes d'hydrodynamique doivent être évoquées, tenant compte notamment des régimes d'écoulement.

Le présent travail a pour but de préciser les conditions de vie sur fond pierreux en eau courante par une analyse hydrodynamique de la microdistribution des larves de simulies.

\section{1. - STATIONS ÉTUdiéES, ESPẼCES, MẼTHOdes}

Plusieurs cours d'eau situés en Vallée d'Aure (Hautes-Pyrénées) ont été observés. 
- Le Nistos vers $500 \mathrm{~m}$ d'allitude présente, dans la zone observée, un lit mineur de $5 \mathrm{~m}$ de large environ. Les rives sont formées d'escarpements rocheux ou d'empierrements consolidés par la végétation. L'écoulement y est tour à tour fluvial et torrentiel sur un fond constitué d'éléments de tailles très variables. Trois espèces de simulies coexistent dans la station étudiée : Odagmia variegata (Meigen), Odagmia obreptans (Edwards), et plus rare, Simulium rupicolum (Seguy et Dorier).

- Le ruisseau d'Aumar forme le déversoir d'une petite tourbière vers $2200 \mathrm{~m}$ d'altitude. Les débits ne dépassent pas $100 \mathrm{l} / \mathrm{s}$. Les passages calmes et rapides alternent avec, dans la partie observée, de faibles chutes de $0,50 \mathrm{~m}$ environ. Le fond comprend des graviers et des pierres atteignant jusqu'à $10 \mathrm{~cm}$ de diamètre. De nombreuses branches et brindilles de pins peuvent fairc obstacle à l'écoulement. Une seule espèce de simulic a été observéc : Odagmia obreptans.

- Le torrent de la Gela, plus profond, est situé vers $1400 \mathrm{~m}$ d'altitude. Dans les passages rapides, souvent étroits et profonds, des blocs immergés atteignent jusqu'à $1 \mathrm{~m}$ de diamètre. Les cascades sont violentes, l'eau est très aéréc. Une seule espèce de simulie a été observée : Simulium rupicolum.

- Le ruisseau d'Espiaube a élé étudié vers $800 \mathrm{~m}$ d'altitude en aval d'une retenue dont les lâchures peuvent entraîner de brusques variations de débit. Pour une pente moyenne de $15 \%$, le profil en long comprend une série de chutes de 0,10 à $0,15 \mathrm{~m}$ de haut. Des écoulements rapides de faible profondeur recouvrent les rochers. La largeur de 2 à $3 \mathrm{~m}$, se rétrécit par endroits à $0,50 \mathrm{~m}$. Une espèce de simulie a été observée : Odagmia obreptans.

Les observations et photographies ont été faites au moyen d'un endoscope et les mesures de profils de vitesse réalisées à l'aide d'un tube de Pitot constitué par de fines aiguilles hypodermiques.

Les canaux d'expériences immergés dans les cours d'eau comprenaient un radier en ciment auquel était accolées des parois latérales transparentes en plexiglass. La longueur de chaque canal atteignait $1 \mathrm{~m}$ pour une largeur de $5 \mathrm{~cm}$ et une hauteur des parois latérales égale à $10 \mathrm{~cm}$.

\section{2. - MICRODISTRIBUTIONS OBSERVÊES}

Les principales observations ont été faites dans le Nistos.

\section{1. - Conditions générales.}

Nous n'avons pas observé de larves de simulies dans les zones à écoulement général de type fluvial ou infra-critique, mais dans les 
zones à écoulement torrentiel caractérisées par une multitude de micro-écoulements.

Dans ces zones, les pierres du fond étaient diversement colonisées selon leur rugosité, leur taille et leur position par rapport à l'écoulement.

Les conditions générales observées peuvent se résumer ainsi :

1) Les larves se fixent sur les pierres polies et évitent les substrats plus rugueux.

2) De petites populations s'établissent sur des galets de diamètre supérieur à $10 \mathrm{~cm}$ environ, et possédant une certaine stabilité.

3) Dans leur position stabilisée, ces galets (jusqu’à $30 \mathrm{~cm}$ de diamètre) s'appuient les uns sur les autres et présentent leur face la plus longue à l'écoulement, avec une contre-pente généralement faible, et un décrochement à l'aval.

4) Sur les pierres, les filets liquides convergent vers la crête où l'on observe une accélération des vitesses. Ensuite, selon différents paramètres (l'inclinaison du radier notamment) ces filets peuvent décoller de la paroi aval et créer un courant de retour. L'écoulement étant généralement tridimensionnel, avec souvent un débouché latéral, un accroissement relatif des vitesses peut localement apparaître.

5) Selon leur position, les obstacles situés à l'aval d'un galet peuvent avoir une influence sur l'aspect de l'écoulement, les répartitions des vitesses et des pressions (fig. 1).
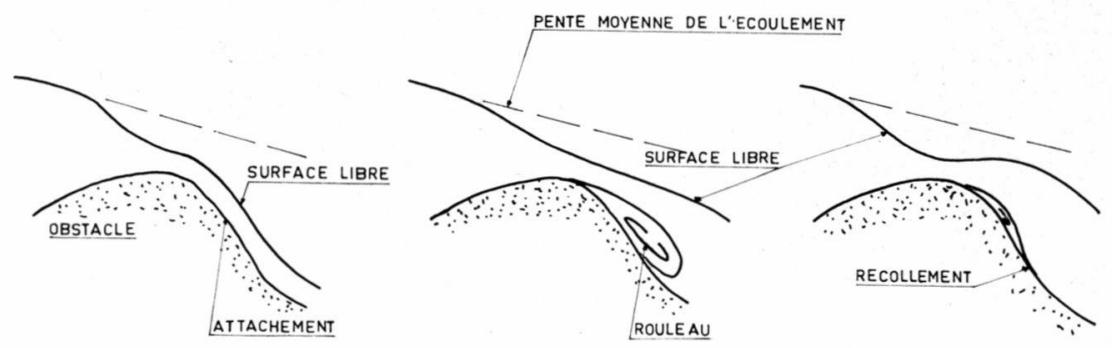

Fig. 1. - Aspects possibles de l'écoulement sur un galet selon la position des pierres situées à l'aval.

\section{2. - Profil en long d'une zone rapide.}

A l'aval immédiat d'une zone où l'écoulement est fluvial avec des vitesses faibles ( $\mathrm{V} \max \simeq 0,20 \mathrm{~m} / \mathrm{s}$ ), et au passage à l'écoulement torrentiel où la vitesse atteint 0,8 à $1 \mathrm{~m} / \mathrm{s}$, on n'observe pas encore de larves de simulies.

Ainsi en est-il de certaines zones où la vitesse atteint $0,6 \mathrm{~m} / \mathrm{s}$ alors qu'un peu plus en aval, le régime torrentiel étant pleinement établi, on observe des larves de simulies pour des vitesses moindres 
$(\mathrm{V} \simeq 0,40 \mathrm{~m} / \mathrm{s})$. Toulefois, dans les zones de transition, cerlaines pierres sont colonisées lorsque de gros rochers provoquent des rétrécissements el une accélération locale des vitesses $(\mathrm{V} \simeq 0.95 \mathrm{~m} / \mathrm{s})$.

Plus en aval, les vitesses maximales observées alleignent $1,50 \mathrm{~m} / \mathrm{s}$. Dans les défilés entre deux pierres, les larves de simulies se répartissent alors fréquemment sur des faces plates, lisses, pour des vitesses uniformes de l'ordre de $1 \mathrm{~m} / \mathrm{s}$. Dans les courants de retour, les vitesses minimales pour lesquelles des larves de simulies sont observées atteignent $0,40 \mathrm{~m} / \mathrm{s}$.

Ces larves sont groupées, solidement fixées à la paroi par leur pseudopode anal. Sous l'effet du couranl, le corps des larves est couché vers l'aval et subit des pulsations de l'ordre de $1 / 10^{\circ}$ de seconde, avec des battements d'environ une demi-seconde et une amplitude voisine de $90^{\circ}$. A l'époque des observations (mai) les groupes de forte densité comprenaient à la fois des larves de simulies et de blépharocérides (photos).

\section{3. - Microdistribution des larves d'Odagmia variegata et $O$. obreptans.}

En règle générale, dans les zones à fort courant, en présence d'un gradient longi!udinal même très réduit des vitesses (sur une pierre plate par exemple) les larves se concentrent dans une étroite bande où les vilesses sont maximales.

\subsection{1. - Conditions de colonisation d'une pierre.}

a) Sur les crêtes, la vitesse d'écoulement atteint souvent son maximum. Ic film laminaire, s’il existe, est très réduit. Par charriage, divers matériaux viennent heurter ces régions : les larves $y$ sont rares.

b) Les faces amont faiblement inclinées présentent les plus fortes densités de larves. En présence d'une incidence réduite et d'une répartition uniforme des vitesses, les larves et nymphes de simulies se distributent sur l'ensemble de la plage offerte à l'écoulement (photo 4).

c) Lorsque les faces frontales sont verticales, la vitesse est très faible au voisinage de la paroi. Quelques rapides transversaux ou obliques peuvent permettre la présence de rares larves de simulies.

d) Les faces aval à décrochement brusque créent des zones de dépression à vitesses pratiquement nulles : elles sont dépourvues de simulies.

e) Les parois latérales, selon leur orientation par rapport à l'écoulement, peuvent abriter de nombreuses larves, concentrées en bancs transversaux, notamment quand la proximité des pierres provoque une accélération des vitesses (photo 1). 

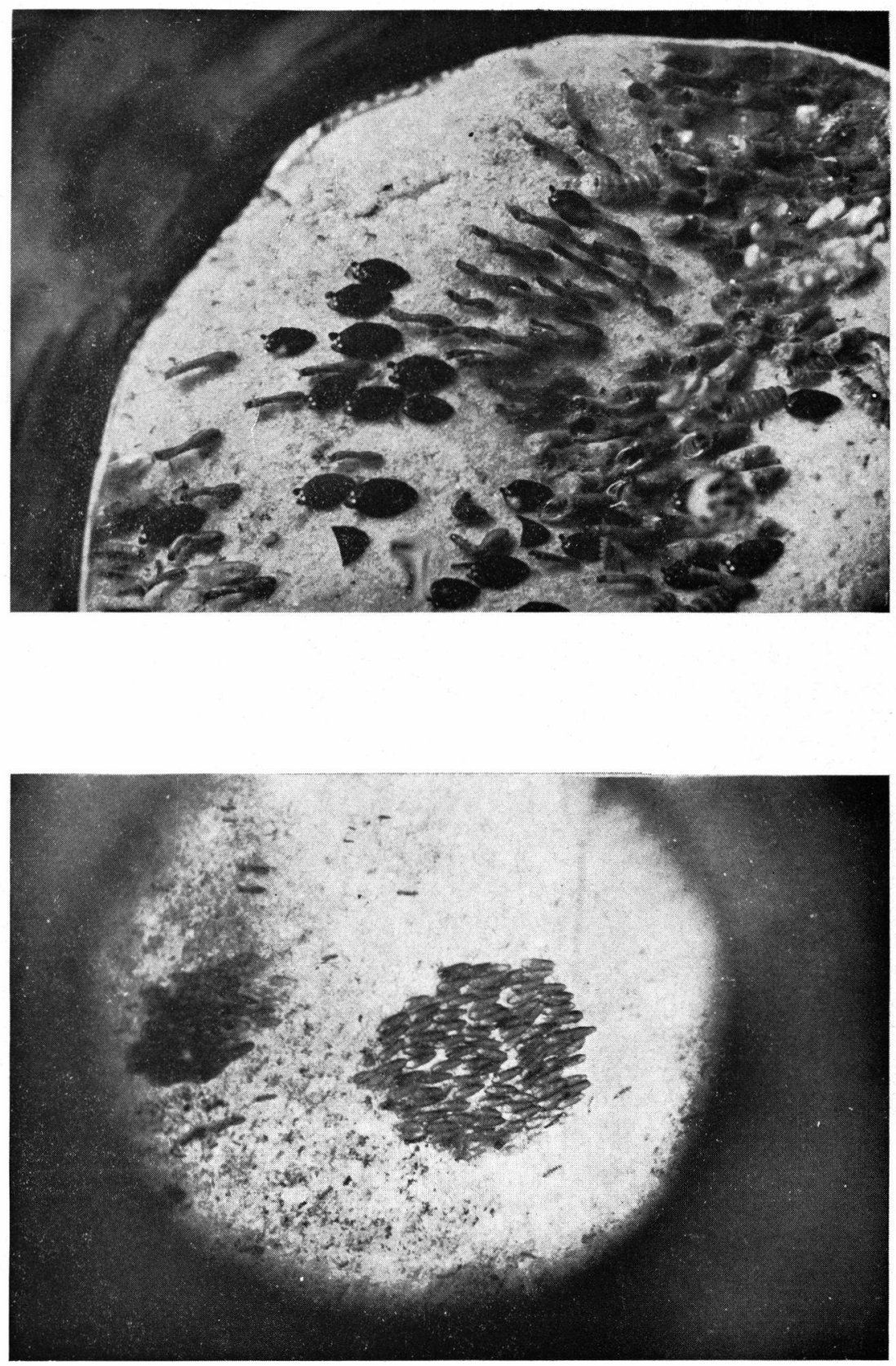

Рното 1. - Larves et nymphes de simulies et blépharocérides dans une zone fortement turbulente.

Рното 2. - Groupes de nymphes de simulies. 

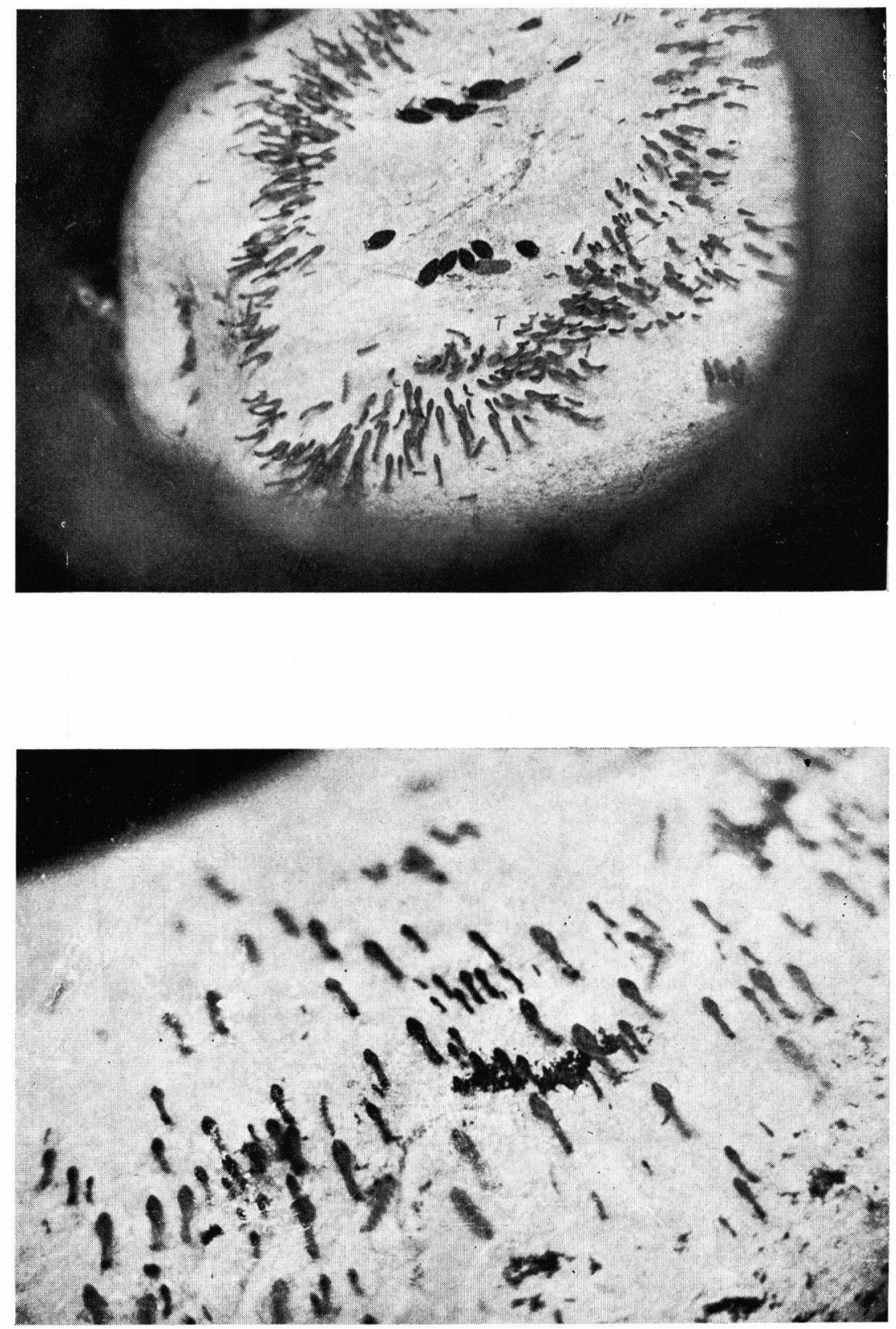

Рното 3. - Larves de simulies réparties concentriquement autour d'un jet frappant une pierre. Les nymphes de blépharocérides sont fixées au point d'impact du jet.

Рното 4. - Larves de simulies en courant uniforme sur une surface plane. 


\subsection{2. - Cas particuliers.}

Certaines configurations présentent de fortes densités de peuplement.

a) A la sortie du couloir formé par deux pierres rapprochées, lorsqu'un jet vient frapper perpendiculairement une face et se divise, les larves se regroupent dans la zone où l'écoulement devient parallèle à la paroi, au niveau où les vitesses sont les plus élevées. Pour un jet sensiblement circulaire, la veine liquide éclate dans toutes les directions el les larves se disposent en are de cercle autour de l'axe central du jet (photo 3 ).

b) Pour certaines pierres, les arêtes rabotées par l'érosion se comportent comme des seuils à profil régulier. En ce cas, les filets suivent la courbure du radier et l'écoulement s'accélère progressivement vers l'aval. De petits accidents de la paroi (aspérités, changements de courbure) peuvent faire décrocher la lame. Un maximum de larves de simulies se trouvent en amont de ces perturbations. Ces larves peuvent également se répartir sur une surface plate recouverte d'une lame d'eau dont l'épaisseur est réduite à quelques millimètres, en écoulement très rapide, parfois légèrement aéré.

\section{4. - Autres observations.}

Selon le tirant d'eau, pour des géométries comparables des pierres, l'écoulement peut avoir des configurations différentes. Par exemple au ruisseau d'Aumar, pour une pente de $5 \mathrm{~cm} / \mathrm{m}$, le lit s'élargit en certains passages avec des vitesses de 0,50 à $0,80 \mathrm{~m} / \mathrm{s}$ et une hauteur d'eau moyenne de $5 \mathbf{c m}$. L'eau s'écoule entre des pierres en relief de $5 \mathrm{~cm}$. Contrairement aux observations précédentes, les larves d'Odagmia obreptans peuplent en ces endroits les faces frontales verticales : étant donné le parcours des filets liquides et la très faible hauteur d'eau, les vitesses y sont élevées.

Les larves sont absentes dans les forles chutes d'une dénivellation de 1 à $4 \mathrm{~m}$, où l'aération est importante. En revanche elles s'accrochent sur les pierres plates en relief dans les cascatelles de 10 à $80 \mathrm{~cm}$ de hauteur, avec des tirants d'eau de 1 à $2 \mathrm{~cm}$ et des vitesses atteignant $1 \mathrm{~m} / \mathrm{s}$.

En eau plus profonde dans le torrent de la Gela, entre 0,50 et $1 \mathrm{~m}$ de profondeur, les larves de Simulium rupicolum ont été observées dans un écoulement aéré, très pulsé, avec des changements brutaux de direction, pour des vitesses variant de 0,80 à $1 \mathrm{~m} / \mathrm{s}$. 


\section{3. - POSSIbilité de COLONISATION DE CANAUX IMMERGÉS}

Sur le terrain, l'étude précise des conditions hydrauliques au voisinage $d u$ fond ne peut se faire que par immersion d'un canal de dimensions connues (voir $\S$ 4.2.1.). La mise en rapport des conditions hydrauliques avec la microdistribution des larves suppose une colonisation possible de ces canaux. Sous cet aspect les larves de simulies présentent l'avantage de s'installer rapidement après la mise en place d'un canal d'expérience (fig. 2).
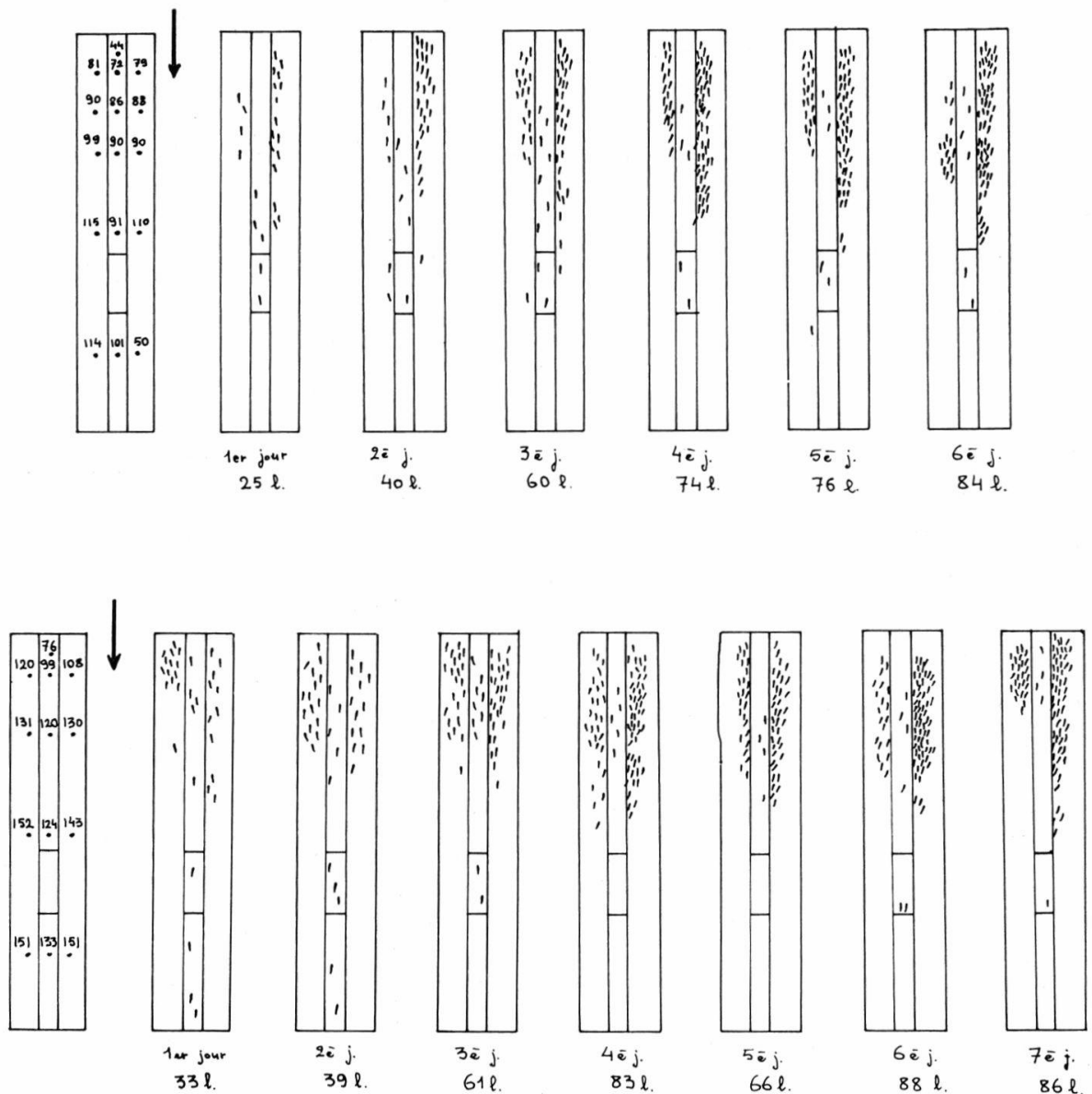

FIg. 2. - Colonisation par Odagmia obreptans d'un canal expérimental placé dans le ruisseau d'Espiaube. Les deux expériences ont été faites au mois d'août : la première jusqu'au $6^{\mathrm{e}}$ jour après la mise en place du canal, la deuxième jusqu'au $7 \mathrm{e}$ jour. Les vitesses de courant ont été prises à l'aide d'un tube de Pitot constitué par de fines aiguilles hypodermiques. Elles sont indiquées à gauche en $\mathrm{cm} / \mathrm{s}$. Pente du canal voisine de $13 \%$ dans chaque expérience. 
Les divers essais réalisés concordent: les larves d'Odagmia obreptans s'accrochent dans la partie antérieure du canal, sur les parois lisses latérales. En ces endroits, les courants ne sont pas les plus rapides (fig. 2) mais la turbulence est réduite par rapport au radier où la présence d'un seuil (partie centrale du canal) provoque des décollements vers l'aval.

La possibilité d'une colonisation rapide des canaux immergés par les larves de simulies permet donc une étude des conditions hydrauliques locales aux endroits précis où s'accrochent ces larves.

\section{4. - RECHERCHE D'UNE ANALYSE HYDRODYNAMIQUE}

Une analyse hydrodynamique de la microdistribution d'invertébrés benthiques doit permettre de répondre aux questions suivantes: Quel est le type d'écoulement dans les stations observées ? Comment varient les profils de vitesse à la paroi ? Dans quelles conditions existe-t-il un film laminaire?

\section{1. - Ecoulement fluvial et torrentiel.}

\subsection{1. - Intérêt.}

L'étude hydrodynamique doit se faire au niveau des larves et porter sur les microécoulements réglant leurs conditions de vie.

Comme le montrent les observations précédentes, les larves de simulies sont situées dans les zones à grande vitesse. Dans les cours d'eau que nous avons étudiés, ces grandes vitesses correspondaient à des tronçons de rivière en écoulements torrentiel. En ces endroits, sur un lit souvent régulier, l'écoulement général n'est plus réglé par le niveau aval mais dépend des conditions physiques locales. Ces conditions ont été étudiées sur le Nistos pris comme exemple de rivière de montagne.

\subsection{2. - Paramètres considérés.}

Dans un tronçon donné de largeur constante, la vitesse moyenne d'écoulement est caractérisée par la valeur du débit et de la hauteur de l'eau.

A débit égal, un régime critique sépare l'écoulement fluvial à faible vitesse de l'écoulement torrentiel à forte vitesse; il est défini, en particulier, par la hauteur d'eau critique.

$$
h_{c}=\sqrt[3]{\bar{q}^{2}} / g
$$

q étant le débit par unité de largeur.

Dans une section, la hauteur d'eau normale dépend du débit q, de la rugosité moyenne $1 / \mathrm{n}$ du substrat, et de la pente longitudinale $\mathbf{i}$ : 


$$
h=\left(\frac{\mathrm{q}}{1 / \mathrm{n} \cdot \mathrm{i}^{1 / 3}}\right)^{3 / 5}
$$

4.1.3. - Types d'écoulement dans une rivière de montagne.

La principale station étudiée sur la rivière le Nistos est située vers $500 \mathrm{~m}$ d'altitude. En cet endroit la surface libre présente une

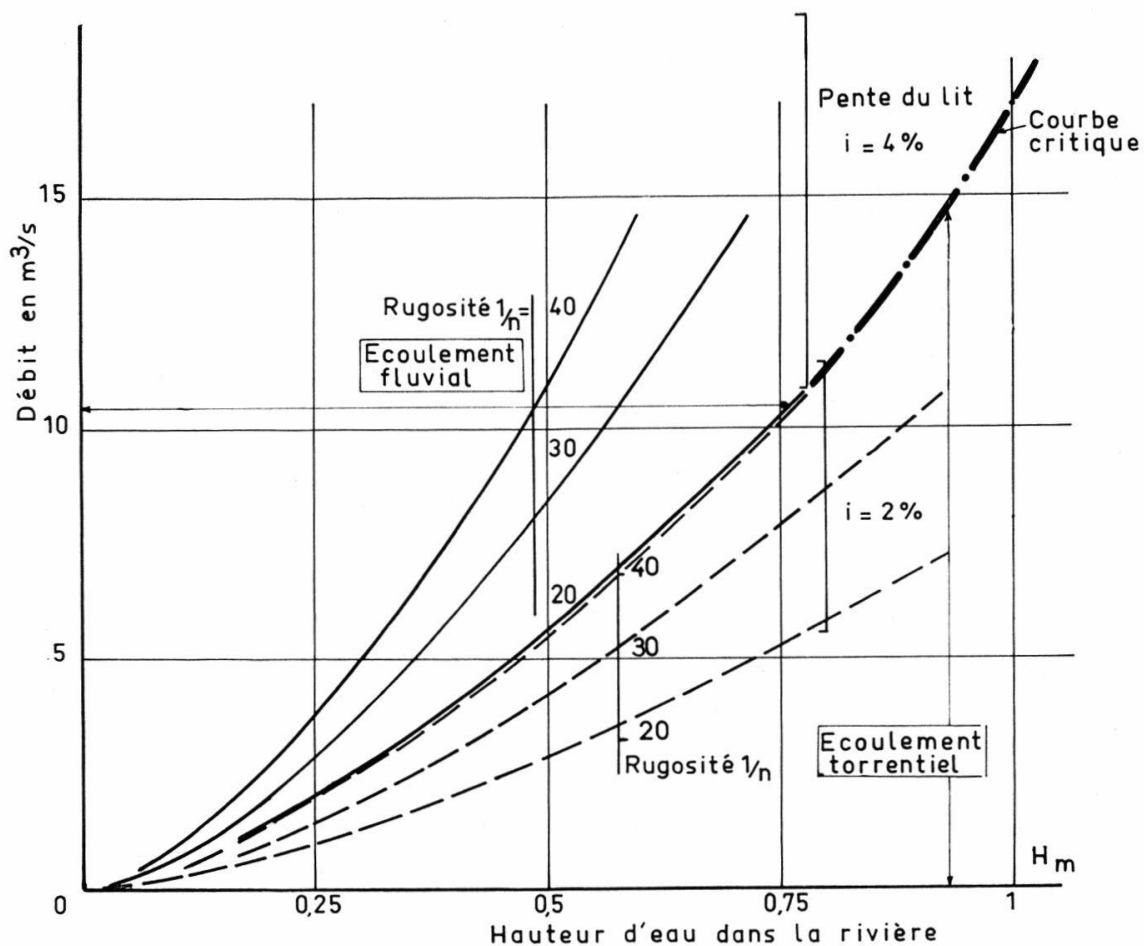

Fig. 3. - Hauteur d'eau marquant le passage de l'écoulement fluvial au torrenitiel en fonction du débit $Q$ pour les rugosités $1 / \mathrm{n}$ variant de 20 à 40 et pour des pentes i égales à $2 \%$ et $4 \%$.

succession de passages calmes à écoulement fluvial et de passages rapides à écoulement torrentiel. La pente des diverses zones, la granulométrie des matériaux, la dimension des aspérités sont très diversifiées dans des tronçons très réguliers.

Pour chacun des paramètres de la formule (2) - hauteur d'eau, débit, rugosité et pente - les valeurs rencontrées dans le Nistos ont servi à tracer les courbes des figures 3 et 4 . En ce qui concerne les rugosités, précisons qu'une valeur $1 / \mathrm{n}=20$ correspond à une rugosité moyenne normale dans une rivière de montagne et que $1 / \mathrm{n}=40$ correspond à un lit de faible rugosité. 


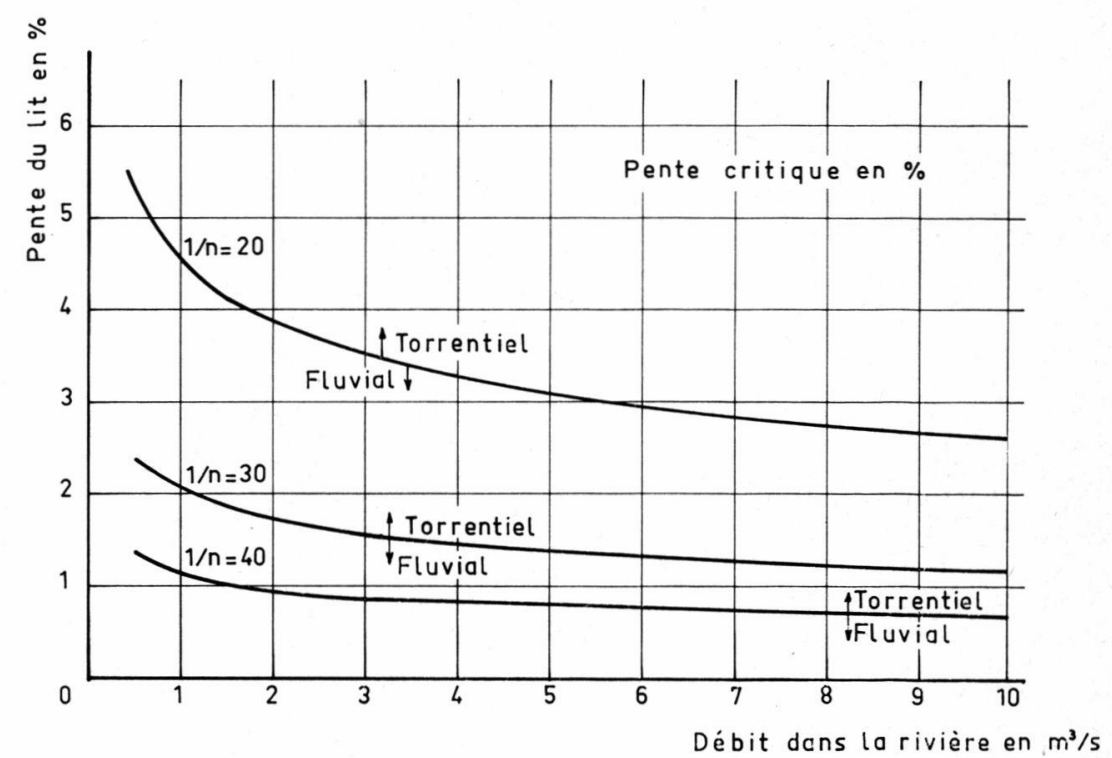

Fig. 4. - Pente critique du passage de l'écoulement fluvial au torrentiel en fonction du débit pour trois valeurs de rugosité.

La figure 3 indique d'une part la courbe de hauteur critique de passage de l'écoulement fluvial (en haut et à gauche) au torrentiel (en bas et à droite) en fonction du débit. Cette même figure indique d'autre part, pour deux pentes $(2 \%$ et $4 \%$ ) et trois rugosités différentes $(40,30$ et 20$)$ les rapports entre le débit et la hauteur d'eau. Ces courbes montrent que pour un débit constan', diverses hauteurs d'eau (et par suite diverses vitesses) peuvent être atteintes selon la pente et la rugosité. Ainsi, dans le même lit du Nistos, pour un débit de $5 \mathrm{~m}^{3} / \mathrm{s}$, h peut varier de 0,30 à $0,75 \mathrm{~m}$, c'est-àdire de part et d'autre de la hauteur critique $0,47 \mathrm{~m}$.

La figure 4 indique pour trois rugosités différentes le passage de l'écoulement fluvial au torrentiel (fortes vitesses) en fonction de la pente et du débit. En effet, la largeur étant constante, la pente du lit se présente sous la forme

$$
\mathrm{i}=\mathrm{k}\left(\frac{\mathbf{1}}{\mathrm{n}}\right)^{2} \mathrm{Q}^{-2 / 9}
$$

Ceci permet de connaître la pente critique pour différentes natures de substrat. Par ailleurs, en un lieu particulier, de pente $\mathrm{i}=4 \%$ et de rugosité $1 / \mathrm{n}=20$, la valeur du débit déterminera le type d'écoulement : torrentiel pour $Q=2,5 \mathrm{~m}^{3} / \mathrm{s}$ et fluvial pour $Q=$ $0,5 \mathrm{~m}^{3} / \mathrm{s}$. 
Ainsi, le débit variant dans le temps en une même station, le régime d'écoulement peut changer et lors du passage en régime fluvial les vilesses peuvent devenir très faibles. De tels changements entraînent très certainement des décrochages des larves de simulies.

\section{2. - Profils de vitesse.}

\subsection{1. - La considération de tranches d'écoulement.}

Les formules et figures du paragraphe précédent permettent de définir la nature de l'écoulement général dans une zone de rivière de montagne telle que le Nistos. Une telle étude est nécessaire mais encore insuffisante pour comprendre la distribution des invertébrés sur le fond. En effet, à une échelle plus précise, de multiples microécoulements apparaissent, chacun ayant sa caractéristique propre. Tous ne peuvent être analysés. Une approche théorique est donc

Il est possible en effet d'isoler certaines branches d'écoulement au niveau même des invertébrés benthiques.

Il est possible en effet d'isoler certaines tranches d'écoulement intéressantes et de considérer les profils de vilesses à la paroi. Ceci peut être réalisé en laboratoire (Ambühl 1959, Trivellato et Décamps 1968, 1971) ou sur le terrain par immersion d'un canal de dimensions connues. Cette tranche d'écoulement schématise l'abord immédiat d'une pierre régulière par exemple. Lorsque la hauteur d'eau est grande, on peut ne considérer qu'une tranche d'écoulement située au niveau où se trouvent les invertébrés, abstraction faite de la surface libre. Les conditions hydrauliques peuvent différer des conditions réelles mais l'erreur ainsi faite demeure négligeable en ce qui concerne la présente étude.

\subsection{2. - Régimes laminaire et turbulent.}

L'étude des profils de vitesse a élé faite par Prandtl et Nikudraze sur conduites circulaires. Nous avons extrapolé leurs résultats à des canaux rectangulaires.

Rappelons que plusieurs régimes d'écoulement peuvent exister dans une conduite. Pour des vitesses très faibles (qui ne nous intéressent pas ici) s'établit un régime laminaire où la viscosité joue un rôle essentiel. Les vitesses augmentant, après une régime de transition s'installe un régime turbulent. Deux lypes distincts se succèdent : en turbulent lisse, la rugosité n'influence pas l'écoulement ; en turbulent rugueux, le coefficient de résistance - ou de perte de charge $\lambda-$, ne dépend plus du nombre de Reynolds. Ces deux types de régimes turbulents onl été rencontrés lors de nos observations. 
Nota. - Il ne faut pas confondre la rugosité au niveau de la paroi dont il est question ici avec la rugosité plus générale traitée au paragraphe 4.1 .

4.2.3. - Analyse des profils de vitesse.

Le profil des vitesses à la paroi dépend de la rugosité, de la vitesse et de la turbulence. Nous avons analysé les profils de vitesse au voisinage de la paroi pour différents substrats, à partir des formules classiques.

a) En turbulent rugueux, l'écoulement correspond à l'égalité

$$
\frac{1}{\sqrt{\lambda}}=2 \log 3,7 \frac{\mathrm{D}}{\mathrm{e}}
$$

$\mathrm{D}=4 \mathrm{R}$ (rayon hydraulique)

$\varepsilon=$ rugosité apparente

$\lambda=$ coefficient de résistance ici indépendant du nombre de Reynolds $\mathscr{R}$, donc de la vitesse et du diamètre.

b) En un point situé à une distance y de la paroi, la vitesse u est donnée par :

$$
\mathrm{u}=\mathrm{U} \cdot\left(8,5+5,75 \log \frac{\mathrm{y}}{\varepsilon}\right)
$$

avec U. (d'après Prandtl) identifié à une vitesse d'échange de turbulence égale à :

$$
\mathrm{U}_{*}=\mathrm{U} \sqrt{\frac{\lambda}{8}}
$$

$$
\mathrm{U}=\text { vitesse moyenne. }
$$

\subsection{4. - Tracé des profils de vitesse.}

La figure 5 indique les profils de vitesse obtenus pour des canaux de dimensions et de rugosités différentes.

Pour mieux comprendre l'influence de la rugosité, on peut comparer les profils indépendamment de la vitesse et des dimensions des canaux (fig. 6). Pour cela, supposons qu'en un point situé à une distance « $d$ » de la paroi, — par exemple $20 \mathrm{~mm}-$, les vitesses soient égales à une même valeur v quelle que soit la vitesse moyenne. Ceci implique que $u_{1}=u_{2}=u_{3}=\ldots$ On obtient alors, pour chaque rugosité apparente $\varepsilon$, des courbes définies et comparables. Nous avons tracé sur la figure 6 les profils pour deux valeurs de $\varepsilon$ : 0,05 (rugosité très faible) et $4 \mathrm{~mm}$ (rugosité forte). La difficulté réside dans le tracé de l'origine de la courbe. Néanmoins, l'influence des aspérités de la paroi apparaît clairement dans la zone où s'accrochent et vivent des larves de simulies par exemple. 


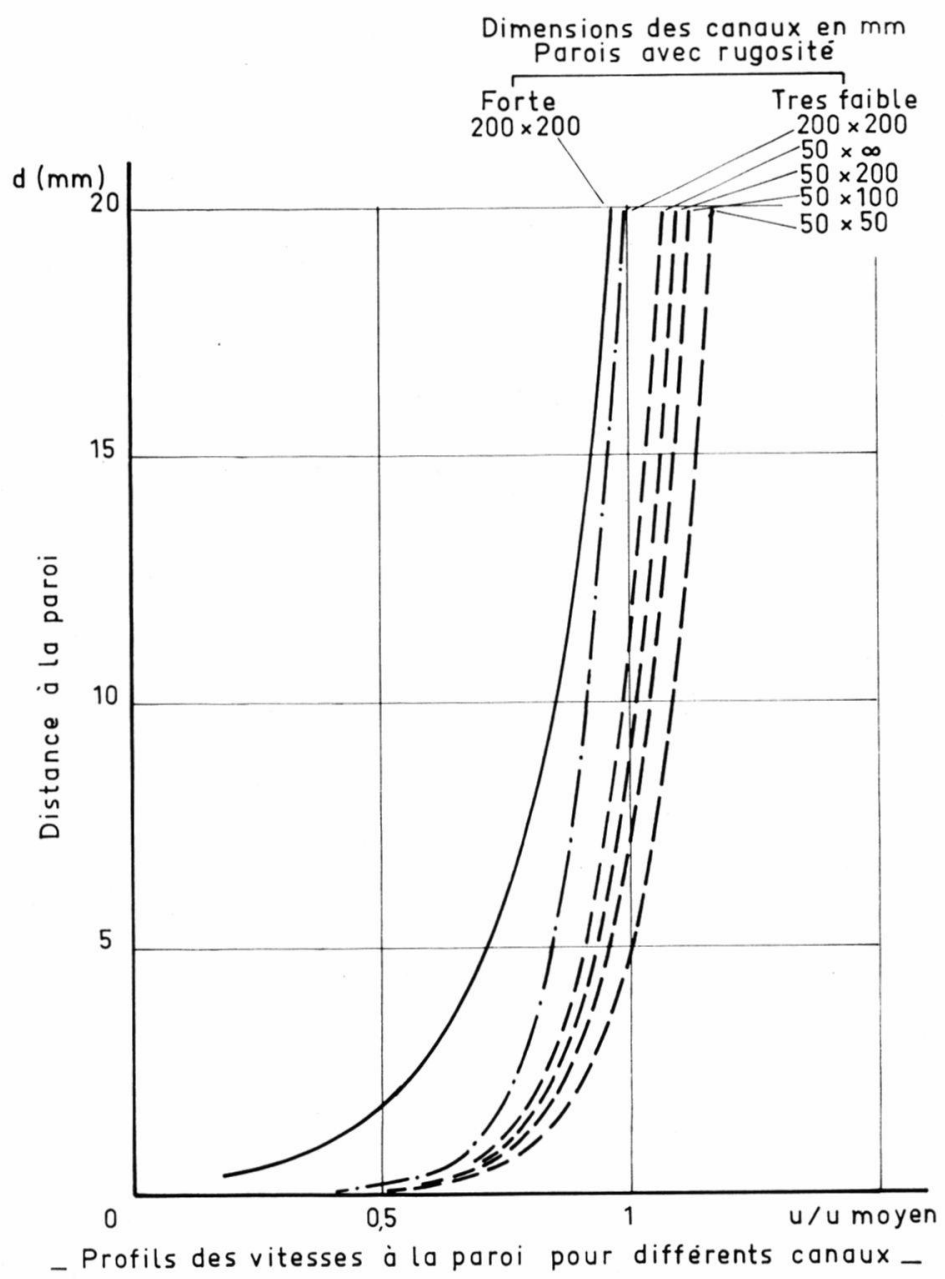

FIG. 5. - Variation des profils de vitesse à la paroi pour des canaux de différentes sections et des rugosités fortes ou très faibles.

\section{3. - Conditions d'existence d'un film laminaire.}

\subsection{1. - Cas d'un canal rectangulaire.}

Selon le régime d'écoulement l'influence des aspérités varie ; il en est de même des vitesses à la paroi et de l'importance du film laminaire. En un même point, des organismes du benthos sont donc soumis à des conditions de vie fort variables. Quel est l'ordre de grandeur de ces variations ?

Théoriquement, le diagramme de Moody permet de connaître en fonction de la rugosité relative $\varepsilon / D$ et du nombre de Reynolds $\mathscr{R}$, la valeur du coefficient de résistance $\lambda$ pour les différents écoule- 
ments : turbulent rugueux, zone de transition, zone critique et écoulement laminaire.

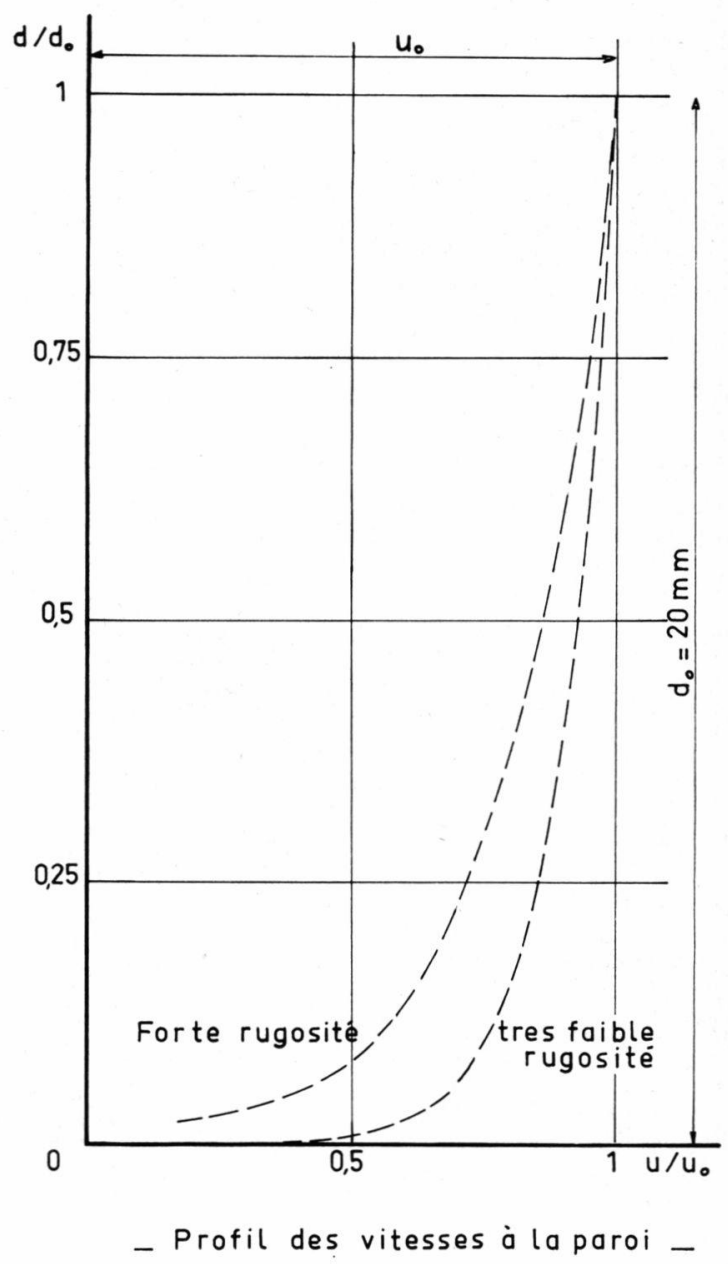

Fıg. 6. - Cas général : Profils des vitesses à la paroi pour deux valeurs de rugosité apparente $\varepsilon=0,05$ (très faible rugosité) et $\varepsilon=4 \mathrm{~mm}$ (forte rugosité).

Dans le cas d'un canal rectangulaire, l'influence du coefficient de forme sur $\lambda$ n'est pas essentielle puisqu'il en est déjà tenu compte dans le calcul de $\mathscr{R}$. Ce facteur de correction, généralement pris sous le radical, a peu d'influence sur les ordres de grandeur recherchés. Par exemple pour un écoulement laminaire, ce facteur brut de correction n'excède pas $\pm 10 \%$ pour un rapport de dimensions de $1 / 1$ à $1 / 3$ dans un canal rectangulaire. 


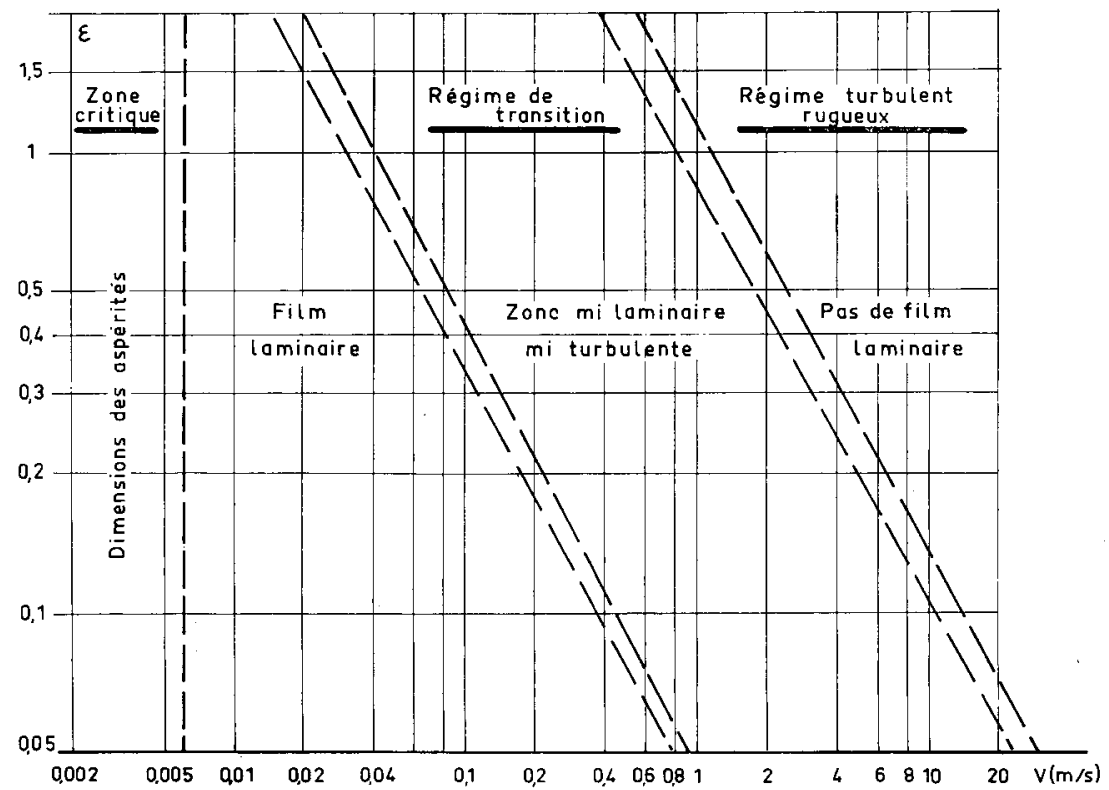

FIG. 7. - Régime établi dans un canal d'expérience en fonction de la vitesse moyenne et de la dimension apparente des aspérités $\varepsilon$.

\subsection{2. - Prévision du régime d'écoulement en canal d'expérience.}

En écoulement uniforme, le régime élabli en canal d'expérience peut être défini en fonction de la vitesse moyenne et de la dimension apparente des aspérités. Les différents régimes possibles sont indiqués sur la figure 7 . Pour un canal (dont les parois ont une très faible rugosité), un régime de transition peut subsister pour des vitesses importantes (par exemple $\mathrm{v} \leqslant 10 \mathrm{~m} / \mathrm{s}$ pour $\varepsilon=0,1$ ) mais dès que les aspérités sont développées, ce même régime de transition n'existe que pour des vitesses ramenées à celles couramment rencontrées dans le courant principal (par exemple $\mathrm{v}<0,2 \mathrm{~m} / \mathrm{s}$ pour $\varepsilon=2$ ). Au-dessus de ces vitesses, le régime est turbulent rugueux sans film laminaire. Rappelons que le diagramme de la figure 7 n'indique pas des frontières rigides entre les régimes d'écoulement mais plutôt des zones critiques de passages.

\subsection{3. - Epaisseur du film laminaire.}

Partant du régime pour lequel existe un film laminaire (fig. 7 ). l'épaisseur de ce film $(\delta=32,5 \mathrm{D} / \mathrm{R} \vee \bar{\lambda})$ diminue rapidement en fonction de la vitesse moyenne aux environs de la zone de transition.

La figure 9 donne une indication concernant l'épaisseur de ce film en fonction de la vitesse. Toutefois, en règle générale, ce film 


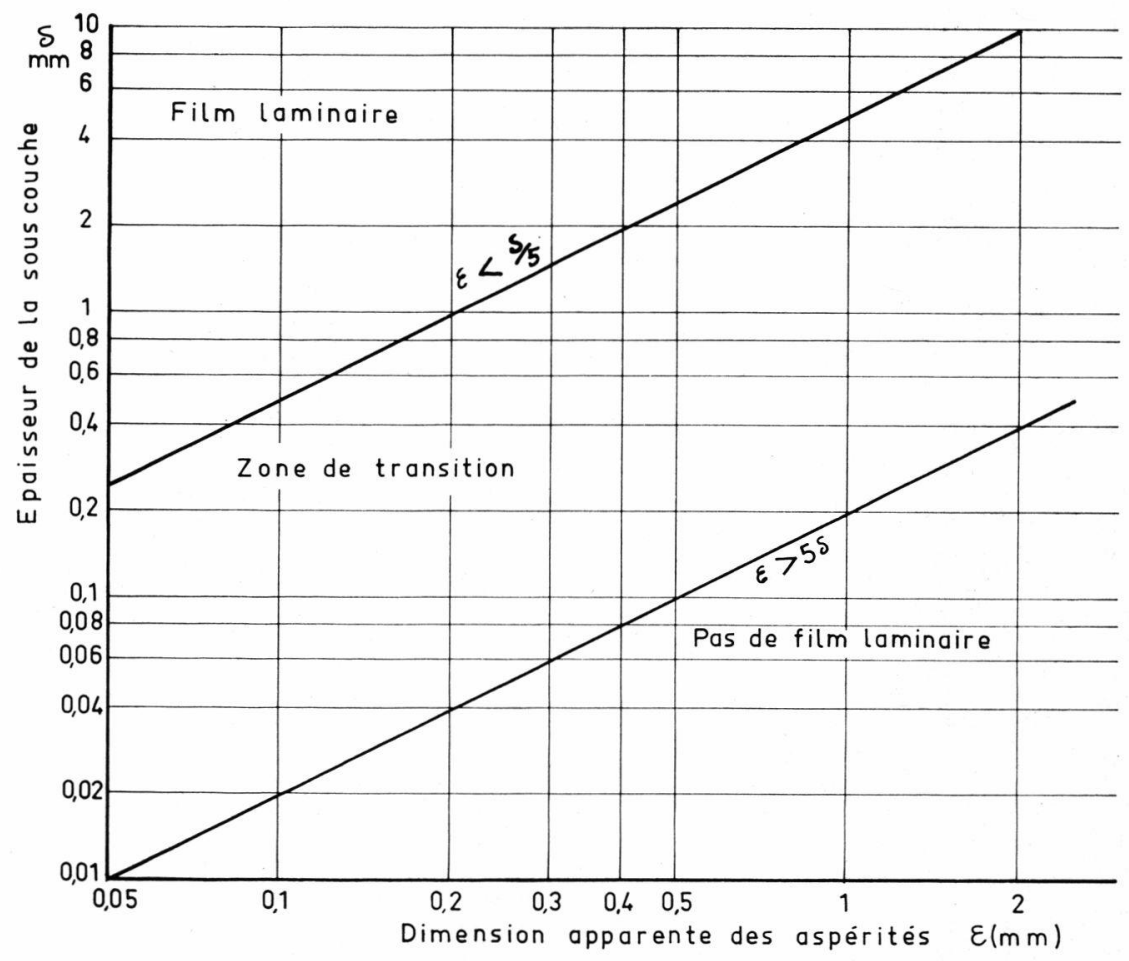

FIg. 8. - Epaisseur de la sous-couche laminaire en fonction de la dimension apparente des aspérités $\varepsilon$.

ne peut exister si $\varepsilon>5 \delta$ mais il est bien défini lorsque $\varepsilon<\delta / 5$. Ces zones sont précisées sur la figure 8.

Il est également tenu compte de cette règle dans la figure 9 dans laquelle ces différentes zones ont été tracées pour quelques valeurs des aspérités $\varepsilon$. Les calculs réalisés pour des sections très différentes ont permis de tracer 2 droites dans lesquelles s'inscrivent les résultats obtenus. Cette figure peut être utilisée comme suit. Pour des aspérités $\varepsilon=2$, l'épaisseur du film laminaire peut varier selon la vitesse de la valeur $\delta=10$ (pour $\mathrm{v} \simeq 0,015 \mathrm{~m} / \mathrm{s})$ à $\delta=0,4$ (pour $\mathrm{v} \simeq 0,5 \mathrm{~m} / \mathrm{s}$ ) ; il n'existera pas dans les zones hachurées, audessus de $\varepsilon=2$ d'une part et à droite de $\varepsilon=2$ d'autre part. Pour des aspérités $\varepsilon=0,5$, la valeur de $\delta$ peut varier, toujours selon la vitesse, de l'épaisseur $\delta=0,25$ (pour $\mathrm{v} \simeq 0,06 \mathrm{~m} / \mathrm{s}$ ) à $\delta=0,1$ (pour $\mathrm{v} \simeq 2,5 \mathrm{~m} / \mathrm{s}$ ) ; il n'existera pas dans les zones hachurées au-dessus de $\varepsilon=0,5$ et à droite de $\varepsilon=0,5$. 


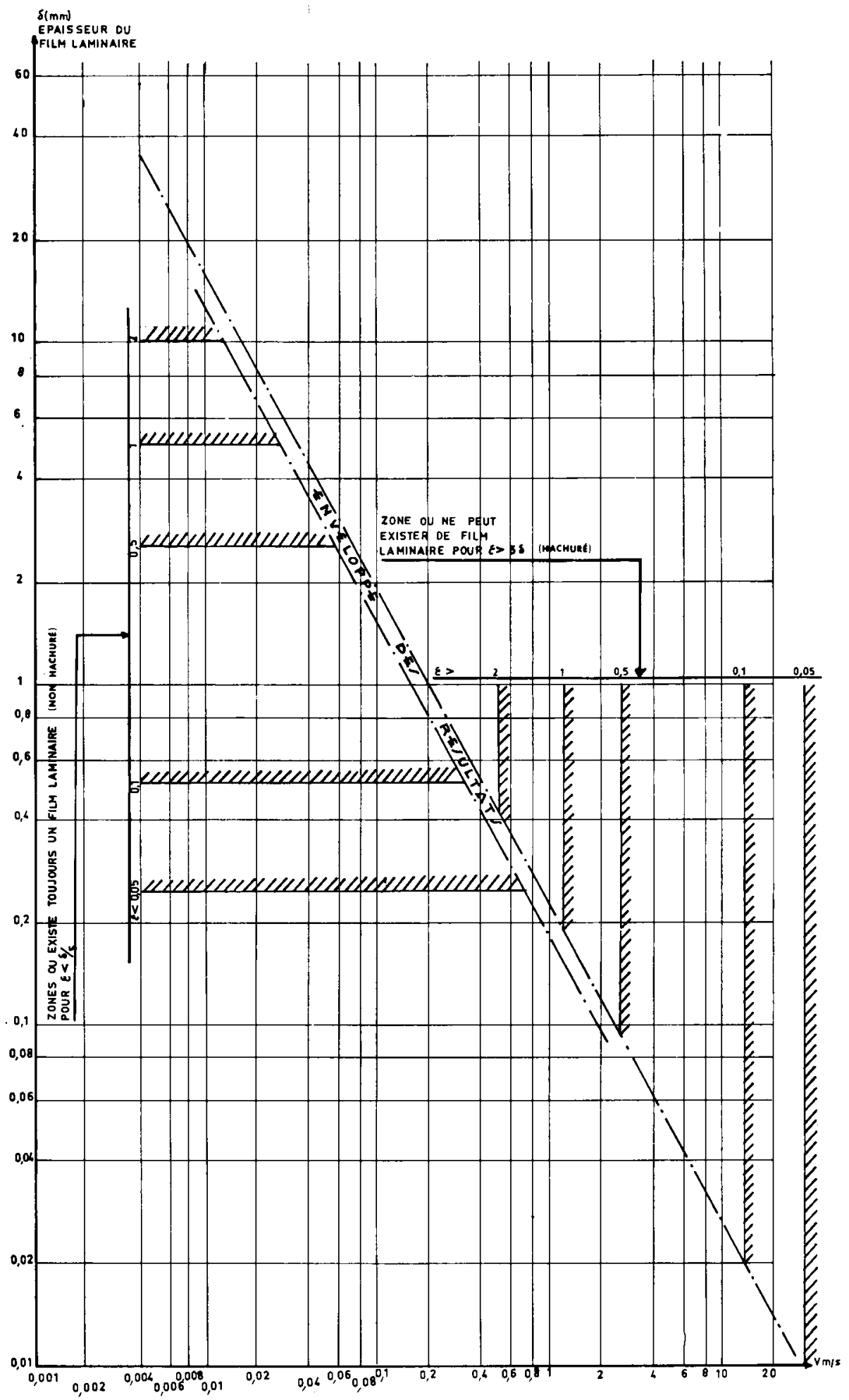

FIg. 9. - Epaisseur du film laminaire ( $\delta$ en $\mathrm{mm}$ ) en fonction de la vitesse ( $v$ en $\mathrm{m} / \mathrm{s}$ ). L'abaque vertical (à gauche) indique les limites des zones où existe toujours un film laminaire (pour une dimension apparente des aspérités $\varepsilon<\delta / 5$ ). L'abaque horizontal (à droite) indique les limites des zones où ne peut exister de film laminaire (pour une dimension des aspérités $\varepsilon>5 \delta$ ). 


\subsection{4. - Conclusions.}

Les calculs et graphiques précédents montrent dans quelles conditions peut exister une sous-couche laminaire. Selon les caractéristiques du courant, on peut ainsi obtenir un ordre de grandeur de l'épaisseur de cette sous-couche et préciser ainsi son influence sur la vie des invertébrés benthiques.

\section{5. - DISCUSSION}

En 1949, Grenier écrivail : «Je pense que si on pouvait évaluer la vitesse du courant à l'endroit même où se tient l'animal, on éprouverait des surprises comparables à celles qu’ont éprouvées les écologistes spécialisés dans l'étude des microclimats».

Pour beaucoup de biologistes, une première surprise fut d'apprendre l'existence dans certaines conditions d'écoulement d'une couche limite laminaire près du fond (Ambühl 1959). Mais le rôle de cette couche limite a été parfois mal compris (Décamps et al. 1972). Le seul régime d'écoulement pour lequel elle existe est rare dans des conditions naturelles. De plus, il est indispensable de tenir compte de l'instabilité permanente qui, à l'échelle de l'habitat, règne dans les conditions naturelles. Nous avons ainsi montré que des variations de pression s'exercent constamment sur le fond d'un canal (Trivellato et Décamps 1968). De même, observées au travers d'un endoscope, les larves de simulies, accrochées aux pierres par leur pseudopode anal, suivent des vibrations constantes ainsi que nous l'avons noté plus haut. Ces faits, et les mesures effectuées, contredisent toute interprélation du comportement des larves de simulies faisant appel à l'existence d'une couche limite laminaire constante sur le fond des eaux courantes (voir Hynes 1970).

En effet, les trois espèces observées ici recherchent des écoulements à fortes vitesses où la turbulence el l'agitation peuvent être grandes. Sans méconnaître d'autres possibilités de prise de nourriture (Serra-Tosio 1967), on peut penser que la vitesse du courant accroît l'intensilé de filtration des particules entraînées par le courant et qu'une certaine turbulence contribue à meltre ces particules plus facilement à proximité du substrat où sont accrochées les larves. Mais une agitation importante devient difficilement supportable par suite des vibrations qu'elle entraîne. Ainsi dans une rivière de montagne, les larves de simulies tendent-elles à éviter les parois rugueuses et les zones les plus perturbées : les colonies les plus denses s'étendent sur les pierres polies, en présence d'un écoulement parallèle à la paroi.

Les figures 3 à 9 présentent des ordres de grandeur de paramètres — rugosité, pente, vitesse —, influençant l'écoulement au voisinage de la paroi. Elles permettent également de prévoir les 
tendances générales de variation en fonction d'un ou plusieurs de ces paramètres. Il est vrai que les courbes tracées dans ces figures supposent réalisées certaines conditions particulières. Dans la nature on ne trouve que rarement des longueurs rectilignes suffisantes pour une extrapolation des résultats théoriques. On n'observe pratiquement pas non plus de régime permanent : des zones de pulsations, des écoulements intermittents, de fréquence et de valeurs aléatoires modifient constamment le profil moyen des vitesses au voisinage du substrat. L'approche théorique permet cependant de mieux comprendre les conditions de vie en fonction de la vitesse, de la rugosité du substrat, ainsi que le rôle de la turbulence. Elle montre, d'une façon plus générale, que les conditions de vie des invertébrés d'eau courante ne peuvent être comprises sans référence à la rugosité du substrat.

Une larve couchée dans le courant, sur une pierre lisse, en présence d'une vitesse à la paroi de l'ordre de $1 \mathrm{~m} / \mathrm{s}$ doit résister à une force voisine de $0,15 \mathrm{cgr}$ pour un $\mathrm{CX}$ (coefficient de traînée) de 0,3 . Cette force dépasserait $1 \mathrm{cgr}$ pour un redressement du corps de la larve sur sa propre hauteur. Un calcul grossier du couple de redressement donne alors unc valeur supéricure à $2,10 \mathrm{~m}$.kg.force. Ces valeurs sont importantes. Elles rendent compte de la position des larves de simulies qui résistent à une action dynamique moindre lorsqu'elles sont couchées dans le sens du courant. Au cours de nos observations, nous avons souvent rencontré des larves ainsi couchées, suivant les vibrations imposées par la turbulence ; nous ne les avons jamais vues dressées face au courant comme on les représente parfois.

Enfin, la colonisation direcle, par dérive, d'habitats turbulents nécessite un important système de freinage (Wu 1930, Grenier 1949, Rühm 1970, Tarshis et Neil 1970). Pour qu'une larve de simulie dérivant dans un courant de l'ordre de $1 \mathrm{~m} / \mathrm{s}$ s'accroche dans une zone déterminée, sur une distance de $1 \mathrm{~cm}$ par exemple, la puissance de freinage devrait être de l'ordre de $10^{-3} \mathrm{~W}$, ce qui est impensable. Seule l'utilisation d'amarres peut permettre aux larves un freinage progressif même si, par ailleurs, certaines dérives à longue distance peuvent être effectuées sans utilisation d'un fil (Rubtsov 1964).

\section{TRAVAUX GITÉS}

Амвӥнц (Н.). 1959. - Die Bedeutung der Strömung als Oekologischer Faktor. Schweiz. Z. Hydrol., 21 : 133-264.

DitTMaR (H.). 1955. - Ein Sauerlandbach. Untersuchungen an einem Wiesen Mittelgebirgsbach. Arch. Hydrobiol., $50: 305-552$.

Dícamps (H.), Capblanco (J.) et Hirigoyen (J. P.). 1972. - Etude des conditions d'écoulement près du substrat en canal expérimental. Verh. Internat. verein. Limnol,, $18: 718-725$. 
Dorier (A.) et Vailiant (F.). 1955. -- Sur le facteur vitesse du courant. Verh. Internat. verein Limnol. 12 : 593-597.

Giudiceldi (J.). 1968. - Recherches sur le peuplement, l'écologie et la biogéographie d'un réseau hydrographique de la Corse centrale. Thèse Sciences, Aix-Marseille, CNRS. A.O. 2478,437 p.

Grenier (P.). 1949. - Contribution à l'étude biologique des Simuliides de France. Physiologia Comp. Oecol., 1 : 165-330.

Hynes (H. B. N.). 1970. - The ecology of running waters. Liverpool, 555 p.

Phillipson (J.). 1956, - A study of factors determining the distribution of the larvae of the blackfly Simulium ornatum Mg. Bull. ent. Res., $47: 227-238$.

Phillipson (J.). 1957, - The effect of current speed on the distribution of the larvae of the blackflies Simulium variegatum $(\mathrm{Mg})$ and Simulium monticola Fried. (Diptera). Bull. ent. Res., 48: 811-819.

Prandtu (L.). 1952. - The essentials of fluid dynamics. London.

RubTsov (I. A.). 1964. - Mode and range of blackfly (Diptera, Simuliidae) larval migrations. Entomol. Rev. $43: 27-33$.

Rüнm (W.). 1970. - Zur Dispersion der Larvenstadien und des Puppenstadiums von Boophtora erythrocephala de Geer (Simuliidae). Z. Angew. Entomol., 6 (3) : 311-21.

Serra-Tosio (B.). 1967. -- La prise de nourriture chez la larve de Prosimulium inflatum Davies 1957 (Diptera, Simuliidae). Trav. Lab. Hydrobiol., 57/58:97-103.

Tarshis (I. B.) et NeiL (W.). 1970. - Mass movement of blackfly larvae on silken threads (Diptera: Simuliidae). Ann. Entomol. Soc. Amer., $63: 607-610$.

Trivellato (D.) et DÉcamps (H.). 1968. - Influence de quelques obstacles simples sur l'écoulement dans un ruisseau expérimental. Annls Limnol., 4 : 357-386.

Trivellato (D.) et Décamps (H.). 1970. - Dispositif expérimental pour l'étude du comportement d'espèces benthiques en eau courante. Annls Limnol., 7 : 145-155.

Wu (Y. F.). 1930. - A contribution to the biology of Simulium (Diptera). Papers Mich. Acad. Sci., $13: 543-99$. 